\title{
Moving Window Principal Component Analysis for Detecting Positional Fluctuation of Spectral Changes
}

\author{
Soo Ryeon Ryu, Isao Noda, ${ }^{\dagger}$ and Young Mee Jung* \\ Department of Chemistry, and Institute for Molecular Science and Fusion Technology, Kangwon National University, \\ Chunchon 200-701, Korea. E-mail: ymjung@kangwon.ac.kr \\ ${ }^{\dagger}$ The Procter \& Gamble Company, West Chester, Ohio 45069, USA \\ Received March 16, 2011, Accepted May 27, 2011
}

\begin{abstract}
In this study, we proposed a new promising idea of utilizing moving window principal component analysis (MWPCA) as a sensitive diagnostic tool to detect the presence of peak position shift. In this approach, the moving window is constructed from a small data segment along the wavenumber axis. For each window bound by a narrow wavenumber region, separate PCA analysis was applied. Simulated spectra with complex spectral feature variations were analyzed to explore the possibility of MWPCA technique. This MWPCA-based detection of the peak shift, potentially coupled with 2D correlation analysis to provide additional verification, may offer an attractive solution.
\end{abstract}

Key Words : Moving window principal component analysis (MWPCA), 2D correlation spectroscopy, Peak position shifts, Principal component analysis (PCA)

\section{Introduction}

A peak positional fluctuation of spectral changes has bothered in spectral analysis for a few decades. It is very difficult to detect the peak shift especially when peaks are overlapped with other contributions. Thus, the detection of position-shifting band is of importance not only in twodimensional (2D) correlation analysis ${ }^{1-6}$ but also for many other general applications, such as multivariate calibration ${ }^{7-10}$ problems. The positional fluctuation of spectral feature is fundamentally nonlinear, not obeying the linear BeerLambert law. This will force the calibration model to incorporate a relatively large number of factors, leading to the unstable over-fitting situation. If one can identify the region of peak position shift prior to calibration model building, such over-fitting can be avoided by masking the contribution of the shifting peak to the model. Methods for detection of peak position shift have been reported, ${ }^{11-17}$ but no method solves the problem completely.

We have recently investigated what really means by socalled positional or frequency fluctuation of spectral features by simple application of principal component analysis (PCA) to spectral data. ${ }^{18}$ It was found that the spectral dataset containing a real band frequency shift requires substantially more principal components (PCs) than normal PCA situations to adequately capture the detailed intensity variations arising from the nonlinear effect. This is because the shifting peak cannot be described as a simple linear combination of other peaks located at different frequencies. In contrast, an apparent positional shift of the peak maximum due to the relative intensity changes of overlapped bands requires only a few significant PCs. We have also demonstrated the potential and pitfalls of using various waterfall plots in the detection of the band positional shifts. ${ }^{19}$ 2D correlation spectra clearly show the very characteristic cluster pattern for both band positional shifts and two overlapped bands. However, the cross peak clusters generated by the peak position shift effect can be sometimes confusing and easily misinterpreted, if the shifting peak is overlapped with intensity changes. A simple visual pattern recognition method, such as the detection of so-called clover and butterfly patterns may not work too well for highly overlapped peaks.

In this study, we propose an idea of utilizing moving window principal component analysis (MWPCA) as a sensitive diagnostic tool to detect the presence of peak shift. In MWPCA, the moving window should be constructed from a small data segment not along the usual perturbation or sample axis but along the wavenumber axis. For each window bound by a narrow wavenumber region, one should apply separate PCA analysis. Depending on the position of the window, the number of principal components (PCs) required to adequately describe the spectral intensity changes (i.e., the effective rank of the window) will vary dramatically. This feature can be easily visualized by constructing the Scree plot (log eigenvalue $v s$ number of PC) for individual windows moving along the wavenumber axis. One may arbitrarily set the threshold for the nominal rank of the window to be the number of eigenvalues which are greater than or equal to some fraction of the first eigenvalue. Once the rank of each window is established, one can plot the number of eigenvalues $v s$ the position of the window on the wavenumber axis. This operation is very similar to evolving factor analysis (EFA), ${ }^{20-22}$ except that the wavenumber axis is scanned instead of the perturbation axis. A distinct increase in the effective rank indicates a region of the possible peak shift.

Data Simulation and Processing. The set of spectra used in this study were synthetic spectra. The spectra consist of 
four Lorentzian peaks located at 1140, 1119, 1100, and 1077 $\mathrm{cm}^{-1}$, respectively, with gradual increase or decrease with different rates in each step. There are a total of ten such steps. A band at $1077 \mathrm{~cm}^{-1}$ has a shoulder band at $1080 \mathrm{~cm}^{-1}$ simultaneously shifting in position and decreasing in intensity. A band at $1119 \mathrm{~cm}^{-1}$ is two overlapped bands changing in intensity in opposite directions. The choice of the wavenumber axis is quite arbitrary, and thus, it can be settled for any other spectral variable. The data treatment was performed by use of MATLAB software (The MathWorks Inc.).

2D correlation spectra were calculated using the algorithm based on the numerical method developed by Noda. ${ }^{6} 2 \mathrm{D}$ calculations were done in MATLAB. PCA analysis was performed by PLS_Toolbox Ver. 4.2 (Eigenvector research, Inc.) for MATLAB.

\section{Results and Discussion}

Figure 1 shows the simulated spectra to show the effect of intensity and positional fluctuation of spectral bands. The intensity changes of bands are indicated by arrows. The enlarged spectra of $1117-1120$ and $1070-1084 \mathrm{~cm}^{-1}$ region represent, respectively, the spectral changes of two bands at
1119 and $1077 \mathrm{~cm}^{-1}$. A shoulder band at $1080 \mathrm{~cm}^{-1}$ is gradually shifted along the spectral axis in the direction of the arrow with simultaneous intensity decrease. A band at $1119 \mathrm{~cm}^{-1}$ represents the intensity changes of two highly overlapped bands with fixed band position and relative line shape. One band at $1119.3 \mathrm{~cm}^{-1}$ decreases in the intensity, while the other band at $1119 \mathrm{~cm}^{-1}$ increases, as indicated by the two arrows.

The synchronous and asynchronous 2D correlation spectra from the simulated spectra in Figure 1 are displayed in Figure 2. The synchronous 2D correlation spectrum in 1073$1083 \mathrm{~cm}^{-1}$ region (Figure 2(a)) shows the four-leaf-clover cluster pattern, comprising two auto peaks and two negative cross peaks. The center of the cluster is located near the spectral coordinate corresponding to the peak maximum position of the average spectrum. The corresponding asynchronous 2D correlation spectrum (Figure 2(b)) shows the very characteristic cluster pattern for the case for band position shift, which is well known as the butterfly pattern. The synchronous 2D correlation spectrum in $1117-1121 \mathrm{~cm}^{-1}$ region where two highly overlapped but separate bands change their intensities, one increasing and the other decreasing (Figure 2(c)) shows the four-leaf-clover cluster pattern, comprising two auto peaks and two negative cross
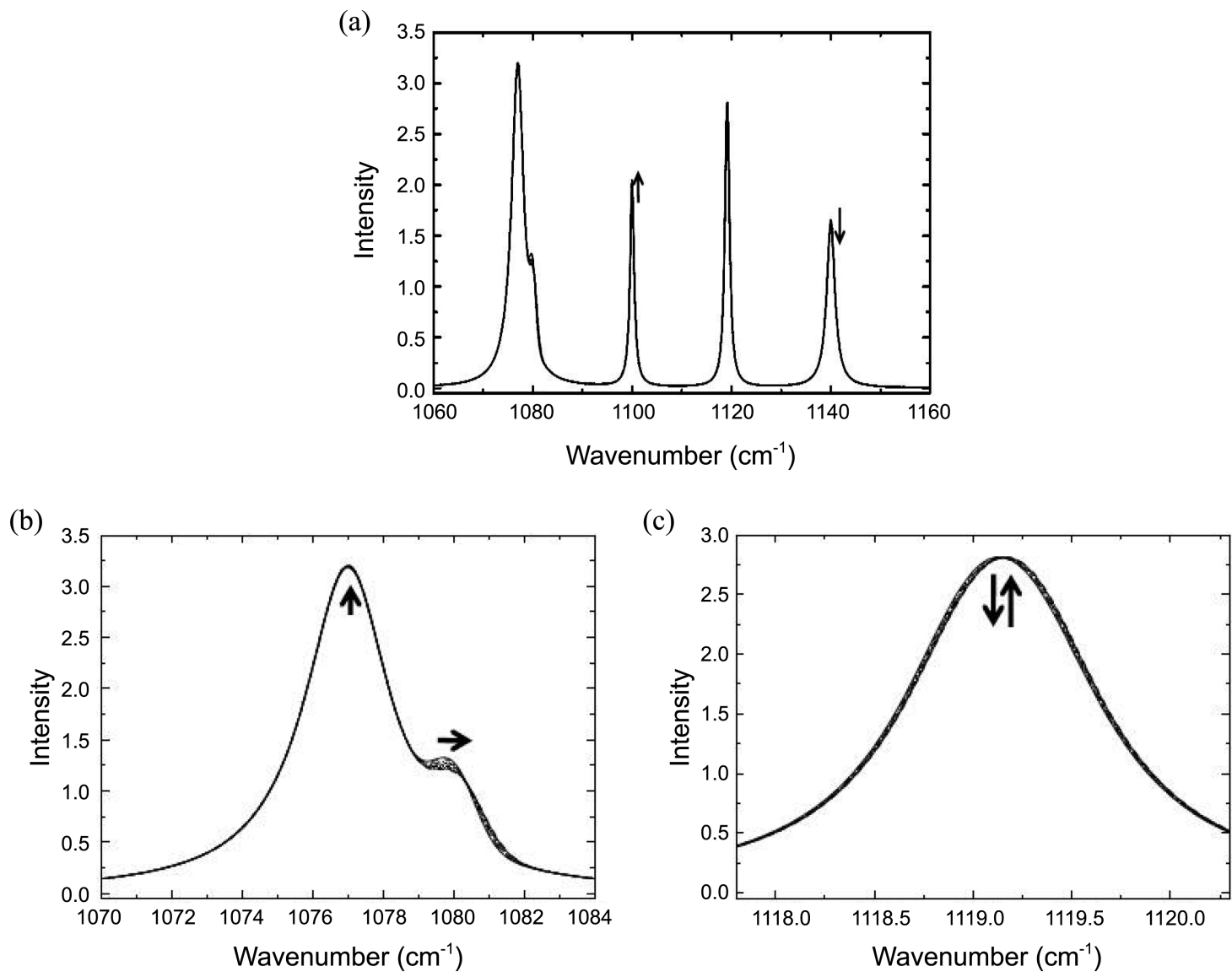

Figure 1. The simulated spectra having four Lorentzian peaks located at $1140,1119,1100$, and $1077 \mathrm{~cm}^{-1}$, respectively, with gradual increase or decrease with four different rates in each step. There are total of 10 such steps. The intensity changes of bands are indicated by arrows. The enlarged spectra of 1117-1121 and 1070-1084 $\mathrm{cm}^{-1}$ region represent, respectively, the spectral changes of two bands at 1119 and $1077 \mathrm{~cm}^{-1}$ 
(a)

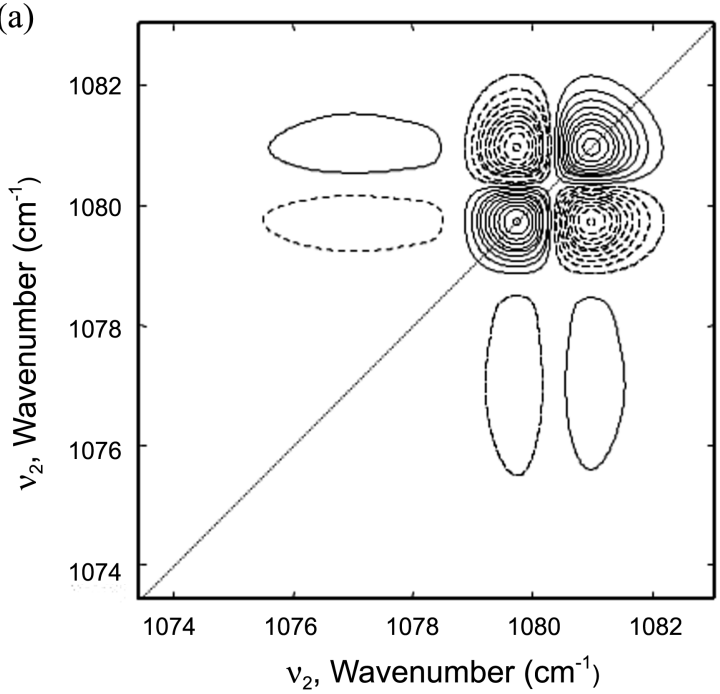

(c)

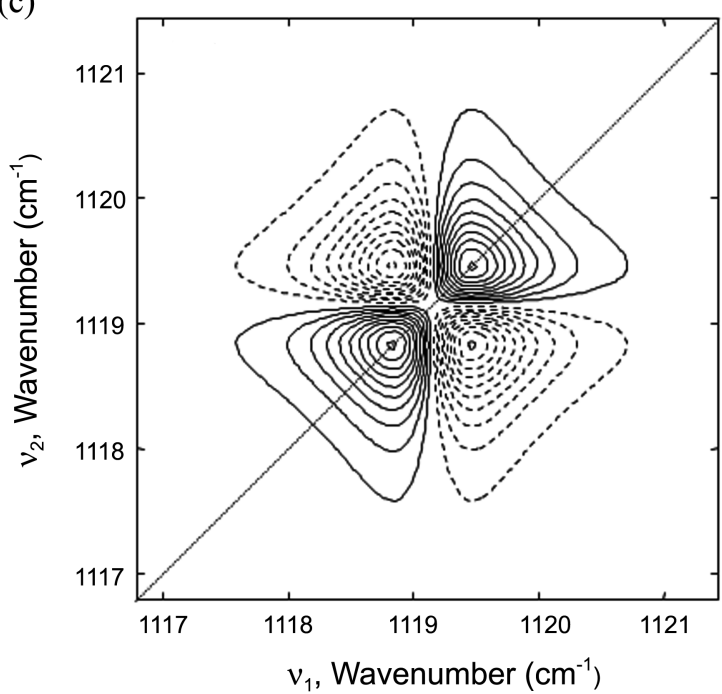

(b)

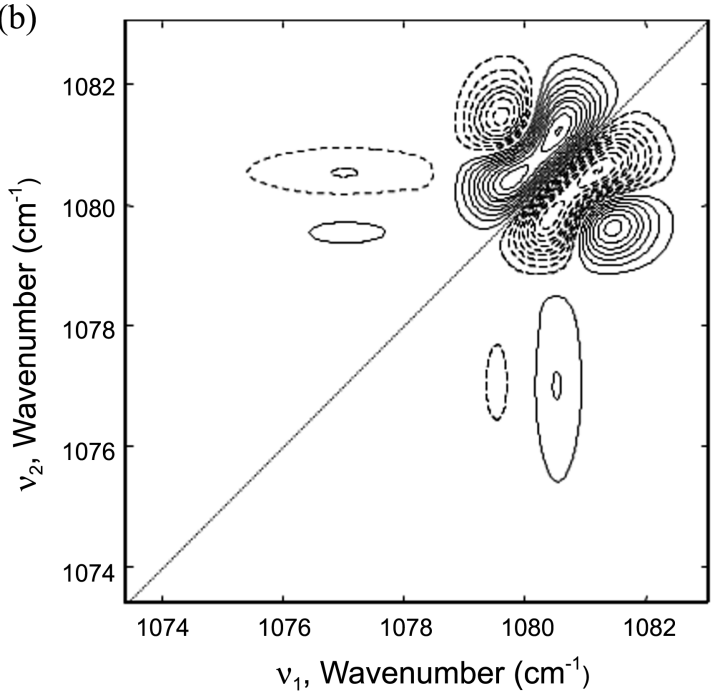

(d)

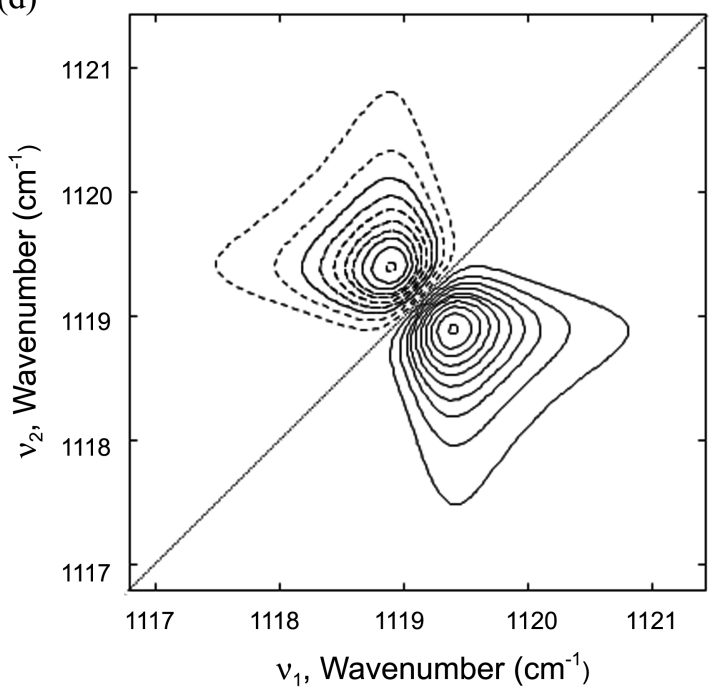

Figure 2. The synchronous and asynchronous 2D correlation spectra from the simulated spectra in Figure 1 for the 1073-1083 (a, b) and $1117-1121 \mathrm{~cm}^{-1}(\mathrm{c}, \mathrm{d})$ region. The solid and dashed lines represent positive and negative cross peaks, respectively.

peaks. The corresponding asynchronous 2D correlation spectrum (Figure 2(d)) shows two well-resolved cross peaks without any further complicating features, which is the very characteristic cluster pattern for the case for two highly overlapped bands.

The plot of loading vectors of PCs for the entire simulated spectra in Figure 1 is shown in Figure 3. PCA factor 1 (PC1), factor 2 (PC2), and factor 3 (PC3), respectively, account for $99.76 \%, 0.23 \%$, and $0.001 \%$ of the total variance of spectral intensities. It means that the spectral intensity variations analyzed over the entire spectral range from 1060 to $1160 \mathrm{~cm}^{-1}$ are dominated by the PC1. This is expected as the structure of the simulated spectra is a relatively simple monotonic change. This result also shows that the "global" PCA encompassing the entire spectral range is usually not sensitive enough to detect any meaningful signs of the isolated peak position shift.

To detect a position-shifting band, we performed MWPCA.

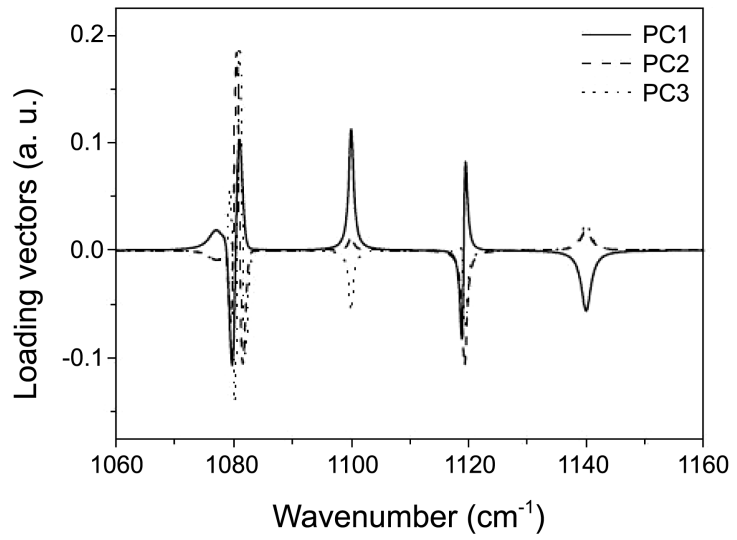

Figure 3. The plots loading vectors of PCs for the simulated spectra in Figure 1. Solid, dashed, dotted lines represent PC1, PC2, $\mathrm{PC} 3$, respectively.

The moving window was constructed from a small data segment $\left(10 \mathrm{~cm}^{-1}\right)$ along the wavenumber axis. For each 
(a)

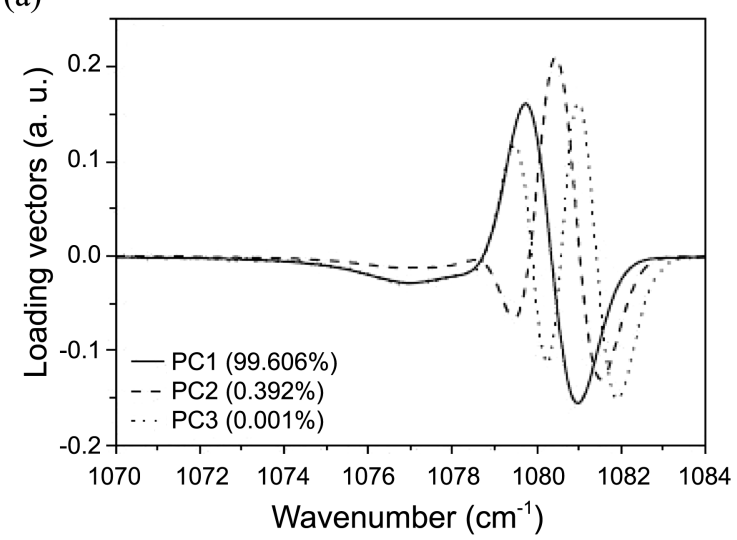

(c)

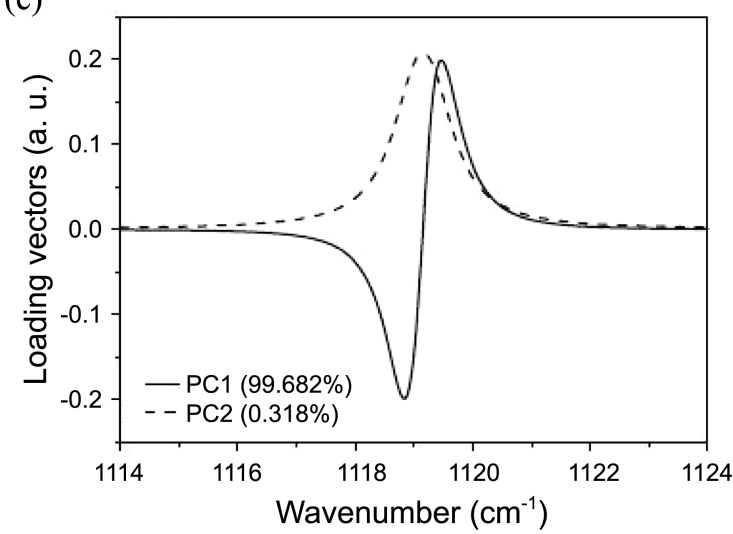

(b)

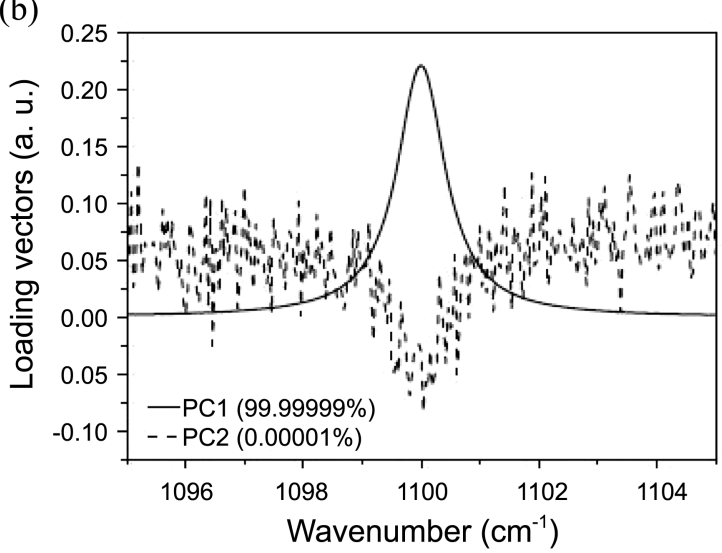

(d)

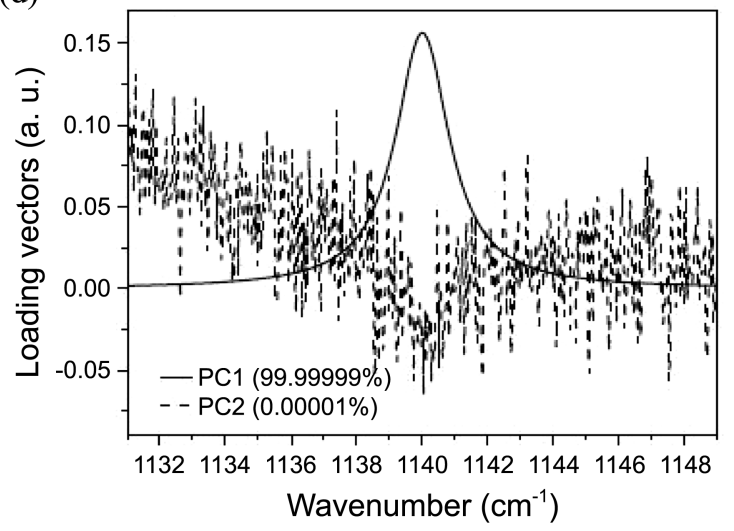

Figure 4. The plots of loading vectors of PCs for four bands regions. Solid, dashed, dotted lines represent PC1, PC2, PC3, respectively.

window bound by a narrow wavenumber region, we applied separate PCA analysis. Figure 4 shows the plots of loading vectors of PCs for four bands regions. For the two bands at 1110 and $1140 \mathrm{~cm}^{-1}$ shown in Figure 4(b) and (d), respectively, within the spectral window between 1095 and 1105 $\mathrm{cm}^{-1}$ and between 1135 and $1145 \mathrm{~cm}^{-1}$, it was found only one $\mathrm{PC}$ is required to model the entire spectral intensity change. For the band at $1119 \mathrm{~cm}^{-1}$ shown in Figure 4(c), the region between 1115 and $1125 \mathrm{~cm}^{-1}$, on the other hand, two significant PCs are required. Therefore, the most of the spectral regions, the total number of required PCs (i.e., the rank of the windowed segment of the dataset) is very small, one or two. In contrast, the region around $1080 \mathrm{~cm}^{-1}$ shown in Figure 4(a) required more PCs to adequately capture the intensity variations. It means that the shifting peak cannot be described as a simple linear combination of a small number of peaks. Loadings in this region look like the derivatives of higher and higher order, and the magnitude of the eigenvalues determined by PCA does not rapidly diminish.

Figure 5 shows the plot of the number of significant PCs (i.e., the matrix "rank" of each window) vs the position of the window on the wavenumber axis. The spectral region between 1114 and $1124 \mathrm{~cm}^{-1}$ has the rank value of 2 , while most other areas are 1 . The region between 1070 and 1084 $\mathrm{cm}^{-1}$ has much higher rank value of at least 3 or greater. It confirms again that most of the spectral region requires only one PC, while some other region, e.g., between 1114 and

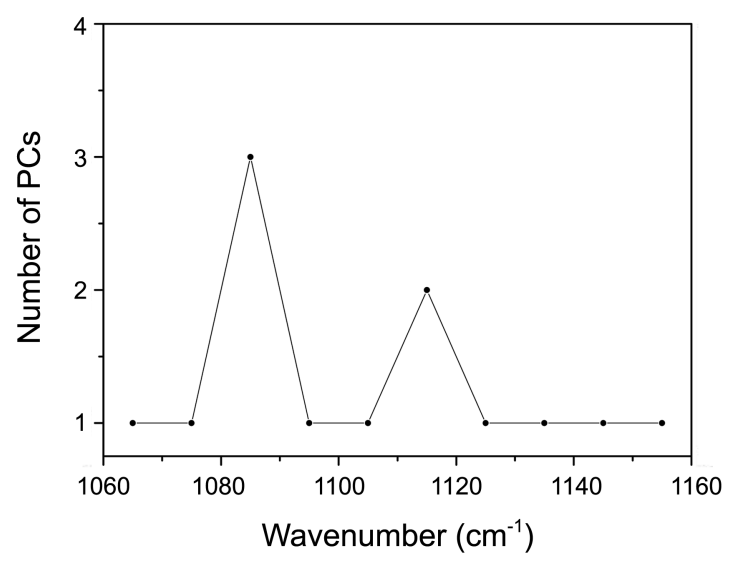

Figure 5. The plot of the number of significant PCs (i.e., the matrix "rank" of each window) vs the position of the window on the wavenumber axis for MWPCA constructed from a small data segment $\left(10 \mathrm{~cm}^{-1}\right)$.

$1124 \mathrm{~cm}^{-1}$, requires at least two PCs. The region of peak shift between 1070 and $1084 \mathrm{~cm}^{-1}$ requires even more PCs. As shown in Figure 4, the loadings for higher PCs change smoothly, indicating that they are probably not noise but real signals. The corresponding eigenvalues for higher PCs is substantially smaller, but the information content is real. Thus, the magnitude of eigenvalue alone may not be a good enough criterion to determine the rank. 

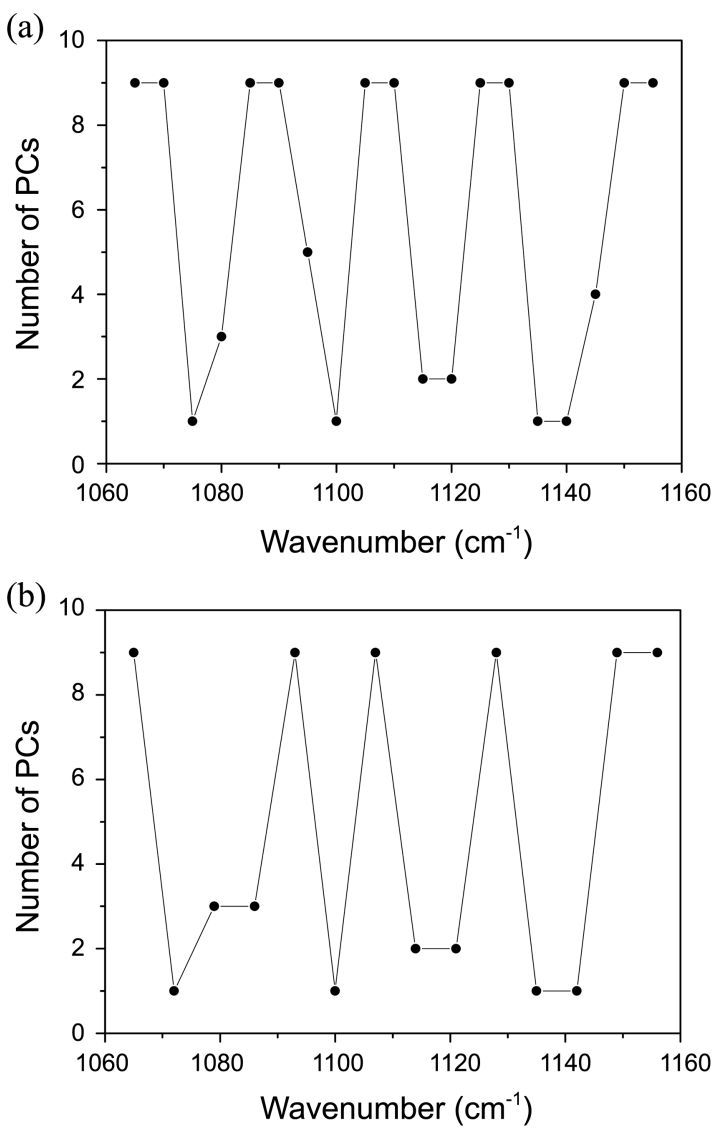

Figure 6. The plot of window rank $v s$ wavenumber for MWPCA constructed from (a) a small data segment $\left(5 \mathrm{~cm}^{-1}\right)$ and (b) a data segment with some overlap of spectral regions with another window $\left(3 \mathrm{~cm}^{-1}\right)$.

To better understand the detection of peak position shift, we performed MWPCA constructed from a small data segment. In this analysis, we moved the position of windows with a finer increment $\left(5 \mathrm{~cm}^{-1}\right)$. The MWPCA constructed from a data segment with some overlap of spectral regions with another window $\left(3 \mathrm{~cm}^{-1}\right)$ was also performed. The plots of window rank $v s$ wavenumber for this MWPCA analysis constructed from a small data segment $\left(5 \mathrm{~cm}^{-1}\right)$ and a data segment with some overlap of spectral regions with another window $\left(3 \mathrm{~cm}^{-1}\right)$ are shown in Figure 6(a) and (b), respectively. It shows in the more detailed plot of the window rank $v s$ wavenumber, which should detect the effect of the highly nonlinear peak-shifting region, where the simple linear Beer-Lambert law is invalid. The technique seems like a good diagnostic to detect the presence of peak shift.

However, windows, 1083-1098, 1103-1113, 1123-1133, and $1150-1160 \mathrm{~cm}^{-1}$, for Figure 6(a) and 1083-1098, 1102$1112,1123-1133,1144-1160 \mathrm{~cm}^{-1}$ for Figure 6(b) have shown a long tail of relatively high eigenvalue extending to PC9. For both non-overlapped and overlapped moving window analysis, the first window is for the peak shift region, while the others are for the almost no signal regions with noise being the dominant contributor to the apparent high rank. It is very clear that the size of eigenvalues alone cannot be used as reliable criterion to determine the number of significant PCs. Essentially most of the higher PCs are noise. If the size of the first engenvalue is very small, which is the case when no peak is located within the window, the relative sizes of higher eigenvalues corresponding to noise will be inflated. Therefore, we have to use the pattern of eigenvectors as well to see when they are dominated by noise.

It is often possible to visually examine the loading plot to see whether the information content is real or not. The PC2 in the region between 1095 and 1105 is a good example. However, it will be better if we can avoid the subjective determination of noisy loading by visual inspection. There is a convenient mathematical index called Durbin-Watson statistic, which can detect randomly fluctuation noise from smooth signals. ${ }^{23-25}$ For a set of numbers in a series, such as

Table 1. The Durbin-Watson statistic values $d$ calculated with loading vectors for the simulated spectra

\begin{tabular}{|c|c|c|c|c|c|c|c|c|c|}
\hline \multirow{2}{*}{$\begin{array}{l}\text { Window } \\
\left(\mathrm{cm}^{-1}\right)\end{array}$} & \multicolumn{9}{|c|}{ Durbin-Watson statistic values $d$} \\
\hline & PC1 & PC2 & PC3 & PC4 & PC5 & PC6 & PC7 & PC8 & PC9 \\
\hline $1060-1070$ & 0.0001 & 1.9502 & 2.0108 & & & & & & \\
\hline $1067-1077$ & 0.0003 & 1.8811 & 1.9923 & & & & & & \\
\hline $1074-1084$ & 0.0059 & 0.0099 & 0.0159 & 0.3115 & 2.0057 & 1.8290 & 2.1122 & 1.9892 & 2.2087 \\
\hline 1081-1091 & 0.0032 & 0.0315 & 0.0650 & 1.4462 & 2.1975 & 2.0058 & 1.8708 & 1.9625 & 1.9893 \\
\hline $1088-1098$ & 0.0007 & 0.3719 & 2.0130 & & & & & & \\
\hline $1095-1105$ & 0.0030 & 0.4026 & 2.1810 & & & & & & \\
\hline $1102-1112$ & 0.0008 & 0.0835 & 1.8866 & & & & & & \\
\hline $1109-1119$ & 0.0050 & 0.1315 & 1.9404 & & & & & & \\
\hline $1116-1126$ & 0.0156 & 0.0024 & 1.9635 & & & & & & \\
\hline $1123-1133$ & 0.0003 & 0.0152 & 1.8356 & & & & & & \\
\hline $1130-1140$ & 0.0007 & 0.3882 & 2.0264 & & & & & & \\
\hline $1137-1147$ & 0.0007 & 1.4097 & 1.7873 & & & & & & \\
\hline $1144-1154$ & 0.0002 & 2.0375 & 2.0005 & & & & & & \\
\hline $1151-1160$ & 0.0013 & 2.0149 & 1.5779 & & & & & & \\
\hline
\end{tabular}




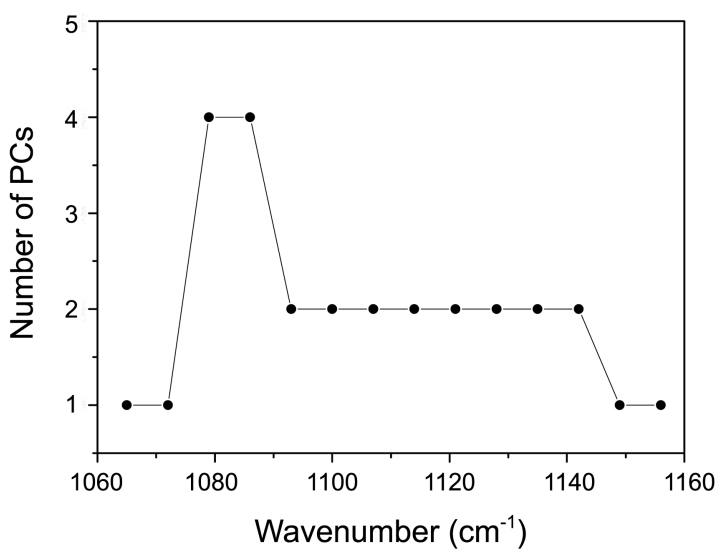

Figure 7. The plot of significant PCs determined by DurbinWatson statistic $d$ values $v s$ wavenumber for the simulated spectra in Figure 1.

a loading vector $\mathbf{v}=\left(\mathrm{v}_{1}, \mathrm{v}_{2}, \ldots, \mathrm{v}_{\mathrm{n}}\right)$, the Durbin-Watson statistic $d$ is defined as: $d=\Sigma\left(\mathrm{v}_{\mathrm{i}}-\mathrm{v}_{\mathrm{i}-1}\right)^{2} / \Sigma \mathrm{v}_{\mathrm{i}}^{2}$. The value of $d$ becomes above some critical value (typically set to about 1.5 or less) if the two adjacent values are not correlated. For purely random noise, $d=2$. Thus, one can use the DurbinWatson statistic to see which loadings are really significant (i.e., low $d$ ) and when the remaining loading becomes noise (high $d$ ). Thus, we can achieve the determination of the rank in a quantitative manner.

We have performed the Durbin-Watson statistic analysis for the simulated spectra to detect the real eigenvectors. Table 1 lists the Durbin-Watson statistic values $d$ calculated with loading vectors (or scores) for the simulated spectra. The Durbin-Watson statistic $d$ values show that only the spectral regions $1074-1084$ and $1081-1091 \mathrm{~cm}^{-1}$ have significant PC3. PC3 for the rest of the spectral regions are all noise, so they have only one or two significant PCs. The number of significant PCs in the two windows, 1074-1084 and 1081-1091 $\mathrm{cm}^{-1}$, have higher PCs, even though the magnitudes of higher eigenvalues are very small. It is obvious that the maximum number of PCS for this simulation was about 4 , located right at the position of the shifting peak. The latter has relatively lower Durbin-Watson statistic $d$ values compared to the rest (even 1080-1090 $\mathrm{cm}^{-1}$ ) for higher PCs. For the windows which have shown a long tail of relatively high eigenvalue extending to PC9, the DurbinWatson statistic $d$ values clearly show that the window for the peak shift region has relatively lower Durbin-Watson statistic $d$ values for higher PCs than the windows for no signal regions with noise being the dominant contributor to the apparent high rank. The plot of significant PCs determined by Durbin-Watson statistic $d$ values $v s$ wavenumber is shown in Figure 7. It looks like a spectrum with flat baseline fluctuating with values of one or two for the entire spectral range, with the exception of sharp spike, around the small region near 1074-1091 $\mathrm{cm}^{-1}$. Thus we can determine if eigenvictors are real or noise-dominated. It clearly shows that Durbin-Watson statistic can reject the inflated rank of small windows where no peak exists.

\section{Conclusion}

We introduced a new and potentially important idea of applying MWPCA technique as a sensitive diagnostic tool to detect the presence of peak position shift. Our results show that the promising potential of detecting of peak position shift by MWPCA analysis. The plot of the number of significant PCs (i.e., the effective matrix "rank" of each window) $v s$ the position of the window on the wavenumber axis shows that the spectral region between 1114 and $1124 \mathrm{~cm}^{-1}$ where two highly overlapped but separate bands change their intensities, one increasing and the other decreasing, has the rank value of 2 , while most other areas are 1 . The region between 1070 and $1084 \mathrm{~cm}^{-1}$ where a band is gradually shifted along the spectral axis has much higher rank value of at least 3 or greater. Durbin-Watson statistic is an excellent diagnostic tool to determine the number of significant PCs, especially for higher order PCs.

This MWPCA-based detection of the peak shift, potentially coupled with 2D correlation analysis to provide additional verification, may offer an attractive solution. We can simply remove the region of peak position shift from the modeling. This might be the simplest approach for detection of peak position shift. Therefore, MWPCA offers a very powerful technique for interpreting highly complicated spectra.

Acknowledgments. This was supported by the Korea Science and Engineering Foundation (KOSEF) grant funded by the Korea government (MOST) (No. 2009-0065428 and 2009-0087013) and the BK 21 program from the Ministry of education, Science and Technology of Korea.

\section{References}

1. Jung, Y. M.; Noda, I. Appl. Spectrosc. Rev. 2006, 41, 515.

2. Noda, I.; Ozaki, Y. Two-Dimensional Correlation Spectroscopy: Applications in Vibrational Spectroscopy; John Wiley \& Sons, Inc.: New York, 2004.

3. Czarnik-Matusewicz, B.; Kim, S. B.; Jung, Y. M. J. Phys. Chem. $B \mathbf{2 0 0 9}, 113,559$.

4. Kim, H. J.; Kim, S. B.; Kim, J. K.; Jung, Y. M. J. Phys. Chem. B 2006, 110, 23123.

5. Noda, I.; Dowrey, A. E.; Marcott, C.; Story, G. M.; Ozaki, Y. Appl. Spectrosc. 2000, 54, 236A.

6. Noda, I. Appl. Spectrosc. 1993, 47, 1329.

7. Malinowski, E. R. Factor Analysis in Chemistry, 2nd ed.; Wiley: New York, 1991.

8. Martens, H.; Næs, T. Multivariate Calibration; John Wiley \& Sons, Inc.: New York, 1991.

9. Vandeginste, B. G. M.; Massart, D. L.; Buydens, L. M. C.; De Jong, S.; Lewi, P. J.; Smeyers-Verbeke, J. Handbook of Chemometrics and Qualimetrics: Part B; Elsevier Science B. V.: Amsterdam, Netherlands, 1998; p 88-104.

10. Brown, S. D.; Sum, S. T.; Despagne, F. Anal. Chem. 1996, 68, 21R.

11. Booksh, K. S.; Stellman, C. M.; Bell, W. C.; Myrick, M. L. Appl. Spectrosc. 1996, 50, 139.

12. Westad, F.; Martens, H. Chemom. Intel. Lab. Syst. 1999, 45, 361.

13. Brown, T. R.; Stoyanova, R. A. J. Mag. Res. B 1996, 112, 32.

14. Siuda, R.; Balecerowska, G.; Aberdam, D. Chemom. Intel. Lab. Syst. 1998, 40, 193. 
15. Witjes, H.; van den Brink, M.; Melssen, W. J.; Buydens, L. M. C. Chemom. Inrtel. Lab. Syst. 2000, 52, 105.

16. Vogt, F.; Booksh, K. Appl. Spectrosc. 2004, 58, 624.

17. Cloarec, O.; Dumas, M. E.; Trygg, J.; Craig, A.; Braton, R. H.; Lindon, J. C.; Nicholson, J. K.; Holmes, E. Anal. Chem. 2005, 77, 517.

18. Ryu, S. R.; Noda, I.; Jung, Y. M. Appl. Spectrosc. 2010, 64, 1017.

19. Ryu, S. R.; Noda, I.; Lee, C.-H.; Lee, P. H.; Hwang, H.; Jung, Y. M. Appl. Spectrosc. 2011, 65(4), 359.
20. Elomaa, M.; Lochmüller, C. H.; Kudrjashova, M.; Kaljurand, M. Thermochm. Acta 2000, 362, 137.

21. Sanchez, F. C.; Rutan, S. C.; Garcia, M. D. G.; Massart, D. L. Chemomet. Intell. Lab. Syst. 1997, 36, 153.

22. Maeder, M. Anal. Chem. 1987, 59, 527.

23. Windig, W. Chemom. Intell. Lab. Syst. 2005, 77, 206.

24. Gourvenec, S.; Massart, D. L.; Rutledge, D. N. Chemom. Intell. Lab. Syst. 2002, 61, 51.

25. Rutledge, D. N.; Barros, A. S. Anal. Chim. Acta 2002, 454, 277. 